\title{
Complements vs. adjuncts and mouse-controlled reading
}

Armine Garibyan

Friedrich-Alexander University Erlangen-Nürnberg, Germany

https://doi.org/10.36505/ExLing-2020/11/0022/000437

\begin{abstract}
The relationship between sentence processing and cognitive demand has received a lot of attention in the past decades. In valency theory, some elements of the sentence are determined by the verbs either in terms of their form or by their presence (Herbst \& Schüller 2008). It has to be said that little attention has been paid to the processing of such fundamental categories in the theory of syntax. On the one hand, this is remarkable since given the amount of research, we still do not know whether this distinction is psychologically real, or whether it only serves a lexicographic and pedagogical purpose. On the other hand, there is a consensus among linguists about the problematic character of the distinction itself even on a more theoretical level (Dowty 2000; Herbst \& Schüller 2008). Therefore, this study attempts to explore whether complements and adjuncts are associated with different kinds of processing. To answer the research questions, an experiment consisting in a mouse-controlled reading task has been designed. To the best of our knowledge, this is a new method in psycholinguistic research. The paper presents the results of a pilot study.
\end{abstract}

Keywords: valency, complements, adjuncts, processing, mouse-controlled reading

\section{Introduction}

In this paper, it has been attempted to uncover whether such theoretical concepts as complements and adjuncts are psycholinguistically valid. The question is topical given the problematic character of such a distinction even on a theoretical level (Hoffmann 2007; Herbst \& Schüller 2008).

A popular and reasonable way to do so would be using an eye-tracker to create a natural setting for reading with items embedded in sentences. However, the costs for eye-tracking experiments are vast, which involves the price of the equipment, laboratory costs, research assistants, etc. In this study, it has been attempted to create a method which would resemble the natural reading process, however with little or no cost - the mouse-controlled reading (MCR).

It is expected that in comparison with adjuncts, complements will present processing advantage given their predictability from the preceding verb. Also, it is believed that those participants with higher level of language proficiency will benefit from this difference the most, given their higher familiarity with these item-specific patterns.

ExLing 2020: Proceedings of 11 th International Conference of Experimental Linguistics, 12-14 October 2020, Athens, Greece 


\section{Experimental setting}

\section{Materials and methods}

The test battery included a personal background questionnaire, a c-test as well as a computer-based experiment (MCR). The c-test was administered as an economical measure of general language proficiency (Klein-Braley 1985). The MCR method consisted in one sentence at a time presented on the screen where the text was always hidden behind (or masked by) a white foreground. By moving the mouse, the participant could see the text behind the mouse ${ }^{1,2}$ (see Figure 1). By limiting the visible region to a small circle, it was possible to tell where exactly the participant was looking at any given point in time. Backtracking was possible, however in terms of one trial only.

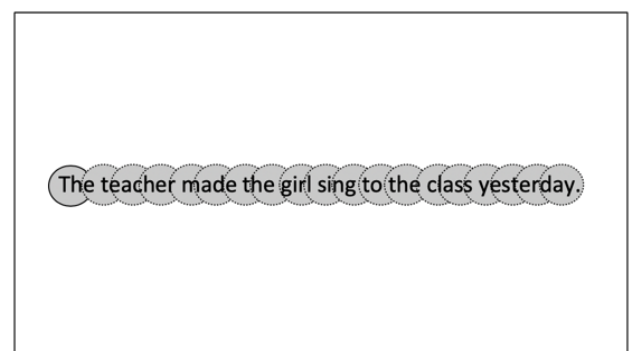

Figure 1. An example sentence of the MCR task. The sentences written on grey background were hidden behind a white foreground. As soon as the reader proceeds from left to right, the previous spot gets covered again.

Participants were asked to read and decide whether the sentence was grammatical or not. Altogether, there were 55 sentences: 15 containing complements, 15 adjuncts and 25 (ungrammatical) fillers for the distractor task. All complement and adjunct units were controlled for length and frequency in the pair and counterbalanced to avoid priming. In (a) and (b) below, the target units are underlined. The verb always appeared in the same word form.

(a) The mother went to search for cigarettes in her son's room.

(b) We can only search with permission on private land.

Being a pilot study, ten participants with L1 German were recruited. The MCR task was presented using the software PsychoPy (version 2020.2.1) on a MacBook Pro 13. Participants' mouse movements were recorded at a sampling rate of $60 \mathrm{~Hz}$ (approximately every $16.6 \mathrm{msec}$ ), i.e. $x$ and $y$ coordinates as well as the time spent at each point. Statistical analysis was carried out in $R$ programming language (R Core Team 2019). 


\section{Results}

A linear mixed-effects model was used to account for individual differences in participants as well as the differences between items. Only target regions (complements and adjuncts) were analysed.

A first inspection of the density plots (Figure 2) shows that participants spent more time reading complements than adjuncts ${ }^{3}$. Although the mean time for reading complement units is $1.92 \mathrm{sec}$ and for adjuncts $2.20 \mathrm{sec}$, statistical analysis has not detected any significant effects $(p=.322)$. Neither has the interaction between condition and language proficiency reached a significance level. However, the analysis showed that responses to the distractor task (i.e. grammatical vs. ungrammatical) were significantly associated with reading times $(p=.002)$, so higher number of correct answers resulted in shorter reading times.
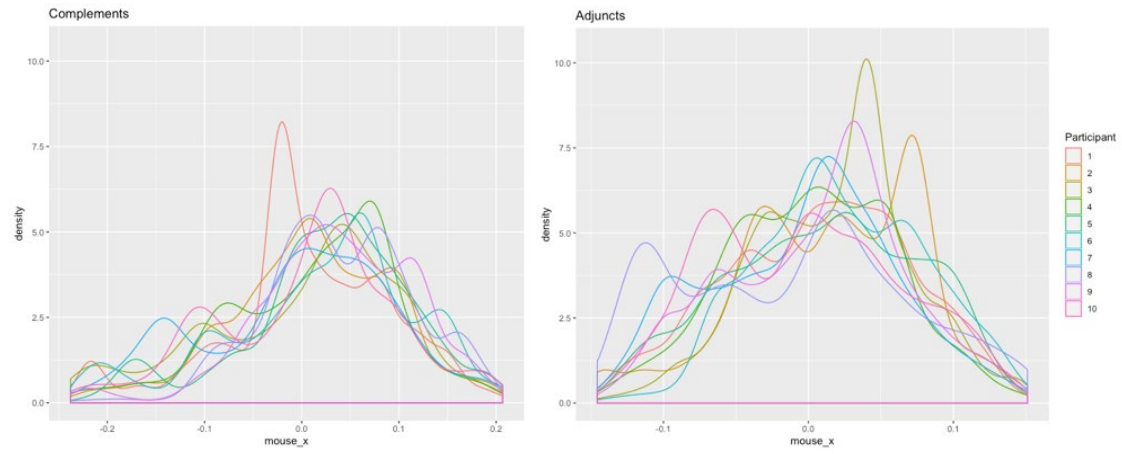

Figure 2. Density plots showing the distribution of data points while reading complement vs. adjunct units by participant.

The findings of the study appear to be in contrast with established literature in the field according to which the predicator entails information on both the argument frame as well as the thematic roles (Rickheit \& Sichelschmidt 2007). What comes next is the reasoning on why the study needs to be continued.

The inspection of Figure 2 allows for further interpretations. First of all, it is clearly visible that there is more alignment between participants' reading flow of the complement units rather than adjuncts, which proves the presence of an unbiased (formal) component predicted by the verb. Second, the general consistency of the reading procedure (in both plots) allows us to think that the MCR should be further studied to explore its potential and validity. Several measures will be taken to continue the study. First, more participants will be tested to reach statistical power. Second, to check whether the absence of the effect on the main condition was caused by the categories themselves or the 
method, a different method will be used to verify the effect. Finally, a different phenomenon will be used in attempt to verify the validity of the MCR method.

\section{Notes}

1. Imagine that you are trying to read a letter in a dark room holding a candle. At each point in time, you can only read the part of the letter at which the candle is pointing.

2. A mouse was chosen over a trackpad to allow for free hand movement (not limited by the size of the trackpad) and thus, a more natural flow of movement.

3. Every point $x$ which the participant passed was associated with $16.6 \mathrm{msec}$ (the refresh rate). The plot shows the frequency of these passings.

\section{Acknowledgements}

I would like to thank Thomas Herbst, Ewa Dabrowska, Peter Uhrig and Laura Becker for their valuable feedback on different parts of the study.

\section{References}

Dowty, D. 2000. The dual analysis of adjuncts/complements in categorial grammar. ZAS Papers in Linguistics 17. 53-78.

Hoffmann, Th. 2007. Complements versus adjuncts: A construction grammar approach of English prepositional phrases. Occasional Papers in Language and Linguistics (University of Nairobi) 3. 92-119.

Herbst, Th. \& Schüller, S. 2008. Introduction to syntactic analysis: A valency approach. Tübingen, Gunter Narr Verlag.

Klein-Braley, C. 1985. A cloze-up on the C-Test: a study in the construct validation of authentic tests. Language Testing 2(1). 76-104.

R Core Team. 2019. R: A language and environment for statistical computing. R Foundation for Statistical Computing, Vienna, Austria. https://www.Rproject.org/.

Rickheit, G. \& Sichelschmidt, L. 2007. Valency and cognition - a notion in transition. In Herbst, Th., Götz-Voteller, K. (eds.), 2007, Valency: theoretical, descriptive and cognitive issues, 163-182. Berlin/New York: Mouton de Gruyter. 